\title{
Hypertension and Hemorrhagic Stroke
}

\section{Sudhir Kumar}

\begin{abstract}
Hemorrhagic strokes are common and account for 20 to $30 \%$ of all strokes in India and the rest of Asia. Patients admitted with intracerebral hemorrhage $(\mathrm{ICH})$ are usually sicker, and $\mathrm{ICH}$ is associated with worse outcomes (as compared with ischemic strokes). Hypertension is the commonest risk factor for spontaneous $\mathrm{ICH}$, responsible for up to $80 \%$ of all cases of $\mathrm{ICH}$, either alone or in combination with other etiological factors. Elevated blood pressure (BP) during the first few hours to days after ICH leads to worse outcomes (higher death and disability rates). This is because high BP is directly linked to hematoma expansion and increased mass effect. Therefore, rapid lowering of BP is recommended if systolic BP > $160 \mathrm{~mm} \mathrm{Hg}$. Intravenous labetalol and nicardipine are both equally safe and effective for this purpose. Survivors of $\mathrm{ICH}$ have a higher risk of developing recurrence of $\mathrm{ICH}$ both over the short term and over the long term. Aggressive BP lowering is recommended in ICH survivors postdischarge from the hospital. The target $\mathrm{BP}$ of $<130 / 80 \mathrm{~mm} \mathrm{Hg}$ should be maintained in them to reduce the risk of $\mathrm{ICH}$ recurrence.
\end{abstract}

Keywords: Antihypertensive agents, Hematoma, Hemorrhagic stroke, Hypertension, Intracerebral hemorrhage, Labetalol, Mortality, Nicardipine, Outcome, Perindopril, Recurrence.

How to cite this article: Kumar S. Hypertension and Hemorrhagic Stroke. Hypertens J 2017;3(2):89-93.

\section{Source of support: Nil}

Conflict of interest: None

\section{INTRODUCTION}

Stroke is a common cause of morbidity and mortality, ranking just behind heart attacks and cancer. Stroke is classified into two types: ischemic and hemorrhagic types. In ischemic strokes, there is blockage of blood supply into an artery, resulting in brain ischemia and infarction. On the contrary, in hemorrhagic strokes, there is rupture of blood vessel, resulting in bleeding into brain parenchyma and damage to brain tissue. Hypertension is the commonest risk factor for nontraumatic hemorrhagic strokes and brain hemorrhage. Other risk factors for hemorrhagic stroke include rupture of an aneurysm, bleeding

\footnotetext{
Senior Consultant

Department of Neurology, Apollo Health City, Hyderabad Telangana, India

Corresponding Author: Sudhir Kumar, Senior Consultant Department of Neurology, Apollo Health City, Hyderabad Telangana, India, Phone: +919866193953, e-mail: drsudhirkumar @yahoo.com
}

diathesis, use of anticoagulants and antiplatelet agents, drug abuse, such as cocaine, etc. The current review article focuses on the relationship between hypertension and hemorrhagic strokes, especially role of hypertension in causing hemorrhagic stroke, clinical features of hypertensive brain hemorrhage, and management of hypertension in patients with hemorrhagic strokes.

\section{Hypertension as a Causative Factor in Hemorrhagic Strokes}

Hypertension has been consistently found as a major risk factor in causing hemorrhagic strokes. In a systemic review, all 14 studies (11 case-control and 3 cohort studies) showed a positive correlation between hypertension and hemorrhagic strokes. ${ }^{1}$ The overall odds ratio of hemorrhagic strokes among hypertensive patients was 3.68. The risk of developing ICH increases with increasing severity of hypertension. Leppälä et $\mathrm{al}^{2}$ found an adjusted relative risk ( $R R_{\text {adjusted }}$ ) of 2.20 for systolic $B P$ of 140 to $159 \mathrm{~mm} \mathrm{Hg}$ and 3.78 for $\geq 160 \mathrm{~mm} \mathrm{Hg}$ compared with $\leq 139 \mathrm{~mm} \mathrm{Hg}$. In another study, Suh et al ${ }^{3}$ found an RR of 2.2 for high normal BP, 5.3 for stage 1 hypertension, 10.4 for stage 2 hypertension, and 33 for stage 3 hypertension. These two cohort studies clearly showed that the higher the degree of hypertension, the higher is the risk of developing $\mathrm{ICH}$.

In a recent study, possible etiologies of ICH were prospectively investigated in 439 patients. ${ }^{4}$ Hypertension was found as an etiology (definite, probable, or possible) in about $80 \%$ of the patients. The next most common etiology was cerebral amyloid angiopathy (in only $30 \%$ of patients). The incidence of hypertension increases with age; therefore, hypertension as a risk factor for ICH is more common in older people. On the contrary, hypertension is not a significant risk factor leading to spontaneous ICH in children. In a recent study, the commonest cause of spontaneous ICH in children was arteriovenous malformation ( $63 \%$ of patients), and hypertension was found in just 1 out of 70 patients. ${ }^{5}$

There are specific mechanisms by which hypertension causes ICH. A high intraluminal pressure in the intracerebral arteries leads to extensive alterations in the smooth muscle wall and endothelium functions. These changes can take the form of hyalinosis, lipohyalinosis (or focal necrosis), and Charcot-Bouchard aneurysms. These degenerative changes in the smooth muscle cells and endothelium predispose to $\mathrm{ICH}^{6}$ 


\section{Locations of Hypertensive ICH}

Intracerebral hemorrhage caused due to hypertension tends to affect specific areas of brain. In a study on 100 patients with hypertensive $\mathrm{ICH}$, the commonest site of bleed was found to be the basal ganglia (55\%), followed by thalamus (26\%), cerebral hemispheres ( $11 \%)$, brainstem $(8 \%)$, and cerebellum $(7 \%){ }^{7}$ In another older case series, Weiner and $\mathrm{Cooper}^{8}$ reported somewhat similar figures. In their series, the locations of hypertensive ICH were as follows: $65 \%$ were in the basal ganglia, $15 \%$ were in the subcortical white matter, $10 \%$ were in the cerebellum, and $10 \%$ were located in the pons.

The reason why hypertensive ICH affects the deeper structures more is because of hemodynamic injury to the perforating arteries, which arise directly from the major arteries and enter the brain at right angles. Cortical vessels have a thicker smooth muscle layer in the tunica media, which protects them from the effects of hypertension. ${ }^{8}$ Perforating arteries on the contrary have thinner walls and are more prone to the effects of high $B P$. The arteries affected include lenticulostriate arteries, thalamoperforate arteries, paramedian branches of the basilar artery, and superior and anterior inferior cerebellar arteries. These arteries supply to basal ganglia, thalamus, pons, and cerebellum respectively, thus explaining the propensity of these areas of brain to be affected more by severe hypertension.

\section{Effects of Hypertension on Acute ICH}

Presence of hypertension influences the short-term and long-term outcomes in patients with ICH. Hypertensionrelated ICH tends to have a higher risk of hematoma expansion, as compared with amyloid angiopathy-related ICH cases. In a recent study, significant hematoma expansion was defined as an absolute increase in the ICH volume $>12.5 \mathrm{~mL}$ or $>50 \%$ within 48 hours of onset. ${ }^{9}$ This study found that significant hematoma expansion occurred in about $45 \%$ of hypertensive patients, as compared with only $19 \%$ of amyloid angiopathy-related ICH patients (odds ratio: 3.081, $\mathrm{p}=0.004$ ).

Hematoma expansion is frequently associated with early neurological deterioration in patients with $\mathrm{ICH}$. In a recent study, neurological deterioration within the first hour (hyperacute) and within 1 to 24 hours (acute) after the onset of ICH was strongly associated with hematoma expansion (odds ratio of 3.6 and 7.6 for hyperacute and acute cases respectively). ${ }^{10}$ In this study, patients with neurological deterioration had worse modified Rankin scores ( 5 vs 3 ; $\mathrm{p}<0.001$ ).

Cerebral edema and perihematomal edema (edema in brain tissue surrounding the hematoma) are independent predictors of poor functional outcome in patients with hypertensive ICH. These lead to increased mass effect and neurological deterioration in patients with $\mathrm{ICH}$. In a recent study, rate of perihematomal edema expansion at 24 hours predicted a higher mortality (odds ratio 2.97) and poor modified Rankin Scale scores (odds ratio 2.21) at 90 days. ${ }^{11}$ Intensive BP lowering within the first 24 hours after the onset of ICH has been shown to reduce cerebral edema and perihematomal edema, leading to better outcomes. ${ }^{12}$

First day mean arterial pressure (MAP) is directly linked to the chance of survival. In a study conducted in Finland, MAP was correlated with chances of survival in 282 patients with supratentorial $\mathrm{ICH}^{13}$ In this study, the MAPs varied between 66.7 and $203.3 \mathrm{~mm} \mathrm{Hg}$, and the cutoff points of the MAP quartiles were 118, 132, and $145 \mathrm{~mm} \mathrm{Hg}$. Patients in the first three quartiles had a fair outcome, with 28 -day survival rates of 71,65 , and $60 \%$ respectively. In contrast, only $33 \%$ of patients in the fourth quartile survived for 28 days $(p=0.001)$. This study also found that comatose/unconscious patients had a higher $\mathrm{MAP}$, as compared with conscious patients. However, at every level of consciousness (alert, somnolent, or unconscious), the proportion of patients dying during 28 days increased from the first to the fourth MAP quartile.

\section{Blood Pressure Control in the Acute Phase of ICH}

Blood pressure lowering is the single most important predictor of better outcome in patients with hypertensive ICH. In hypertensive ICH, we aim for faster and tighter $\mathrm{BP}$ lowering in the acute phase. This is in contrast to acute ischemic stroke, where BP lowering is not considered in the acute phase. This is because lowering of BP may reduce cerebral perfusion pressure, leading to further ischemia and brain damage in patients with acute ischemic stroke.

In a recently conducted study (interact 2), rapid BP lowering in patients with ICH resulted in better functional outcomes. ${ }^{14}$ In this study, about 2,800 patients with spontaneous ICH (within the previous 6 hours) with elevated systolic BP were assigned to two groups: (1) intensive treatment to lower BP to systolic level of $<140$ $\mathrm{mm} \mathrm{Hg}$ within 1 hour; and (2) guideline-recommended treatment with a target systolic BP $<180 \mathrm{~mm} \mathrm{Hg}$. The choice of antihypertensive agent and the dose was left to the treating physician. The group receiving intensive therapy had a significantly lower modified Rankin scores at 90 days (odds ratio 0.87, p = 0.04), as compared with guideline-recommended treatment. Intensive BPlowering group also had better physical and mental health-related quality of life on EQ-5D scale. So, this study indicated improved functional outcomes with intensive lowering of BP. Mortality rates, however, did not differ between the two groups (11.9\% in intensive lowering group vs $12 \%$ in guideline-recommended group). 
In another recently concluded study (ATACH-2), 1,000 patients with ICH (volume $<60 \mathrm{~cm}^{3}$ ) and Glasgow coma scale score of 5 or more with high systolic BP were assigned to intensive treatment (target systolic BP 110-139 $\mathrm{mm} \mathrm{Hg}$ ) or standard treatment (target systolic BP $140-179 \mathrm{~mm} \mathrm{Hg}){ }^{15}$ Intravenous nicardipine was administered within 4.5 hours of symptom onset to achieve the target systolic BP. There was no difference between the two groups in terms of death or disability at 90 days (38.7\% in intensive treatment group versus $37.7 \%$ in standard treatment group).

In a Scandinavian study, the effect of BP lowering with candesartan was assessed in 274 patients with ICH (SCAST trial).${ }^{16}$ Patients with systolic BP $>140 \mathrm{~mm} \mathrm{Hg}$ received candesartan or placebo for 7 days. There was no difference between treatment and placebo groups with respect to the risk of vascular events (death, stroke, or myocardial infarction). Functional outcome tended to be worse in candesartan-treated group.

In another study (CHHIPS trial), patients with ICH or cerebral infarction, with systolic $\mathrm{BP}>160 \mathrm{~mm} \mathrm{Hg}$, were randomly assigned to treatment with lisinopril, labetalol, or placebo, within 36 hours of symptom onset. ${ }^{17}$ Lisinopril and labetalol were found to be safe and effective BP-lowering agents in acute stroke including ICH. Three-month mortality was halved in active treatment group $(9.7 \%$ in active treatment group vs $20.3 \%$ in placebo group). The main drawback of this study was the small sample size and including patients with both ischemic and hemorrhagic strokes.

Many patients are already on antihypertensive medications when they suffer from ICH. One study assessed whether it is advisable to continue or stop the ongoing antihypertensive medications in such patients [Continue or Stop Post-Stroke Antihypertensives Collaborative Study (COSSACS) trial]. ${ }^{18}$ A total of 379 patients were assigned to "continue" and 384 were assigned to "stop" preexisting antihypertensive medications for 2 weeks. This study found that continuation of antihypertensive drugs did not reduce 2-week death, or dependency, cardiovascular event rate, or mortality at 6 months. The drawback of this study was that it was underpowered due to early termination of the trial.

This point-whether to continue or withhold the preexisting antihypertensive medications within the first few days after the onset of $\mathrm{ICH}$ - was assessed in another recently published study-ENOS trial. ${ }^{19}$ About 4,000 patients were enrolled and randomized to receive 7-day treatment of transdermal glyceryl trinitrate $(5 \mathrm{mg}$ per day) or no glyceryl trinitrate. Further, about 1,000 were asked to continue their preexisting antihypertensive medications, and about 1,000 were asked to stop their preexisting antihypertensive medications. Significantly lower BP was seen in groups receiving glyceryl trinitrate as well as those continuing their preexisting antihypertensive medications; however, no improvement in functional outcome was noted in either of the groups. This study concluded that there is no evidence to support continuing prestroke antihypertensive drugs in first few days after $\mathrm{ICH}$.

\section{Guidelines of the AHA/American Stroke Association for the Management of Hypertension in Acute Spontaneous $\mathrm{ICH}^{20}$}

- For ICH patients presenting with systolic BP between 150 and $220 \mathrm{~mm} \mathrm{Hg}$ and without contraindication to acute BP treatment, acute lowering of systolic BP to $140 \mathrm{~mm} \mathrm{Hg}$ is safe (Class I; Level of Evidence A) and can be effective for improving functional outcome (Class IIa; Level of Evidence B).

- For ICH patients presenting with systolic BP > $220 \mathrm{~mm} \mathrm{Hg}$, it may be reasonable to consider aggressive reduction of $\mathrm{BP}$ with a continuous intravenous infusion and frequent BP monitoring (Class IIb; Level of Evidence C).

\section{Role of Hypertension in causing Recurrence of Hemorrhagic Stroke}

Patients with hypertensive ICH have a high risk of recurrence. In a recently published study from Denmark, 15,270 patients diagnosed with primary ICH were followed up for 5 years. ${ }^{21}$ The recurrence of ICH was noted in $8.9 \%$ patients after 1 year and 13.7\% after 5 years. Surgical treatment and renal insufficiency were associated with increased recurrence risks of ICH (RR 1.64 and 1.72 respectively), whereas treatment with antihypertensive medications was associated with reduced risk of $\mathrm{ICH}$ recurrence (RR 0.84). This study concluded that patients with ICH warrant an aggressive management of hypertension to prevent short-term and long-term recurrence of ICH, especially in those treated surgically for primary $\mathrm{ICH}$ and patients with renal insufficiency.

In another single-center study from Boston (USA), 1145 patients with ICH were followed up at regular intervals for a median duration of 36.8 months (minimum 9.8 months). ${ }^{22}$ Recurrence of ICH was noted in 102 of 505 $(20 \%)$ survivors in lobar ICH, and 44 of 640 (6.9\%) survivors of non-lobar ICH. The recurrence rate of both lobar and nonlobar ICH was found to be higher in patients with inadequate control of BP. Therefore, stricter BP control is needed in ICH survivors to prevent its recurrence.

\section{Choice of Antihypertensive Agent in Acute ICH}

There is a lack of published data regarding the specific antihypertensive agent to be used in the setting of acute 
ICH. In a recent survey conducted in Japan, most neurologists and neurosurgeons preferred using intravenous nicardipine for lowering BP in patients with acute $\mathrm{ICH}^{23}$ In a recent study, the efficacy and safety of nicardipine and labetalol infusion were compared. ${ }^{24}$ Eighty-one patients with ICH or subarachnoid hemorrhage, who received either nicardipine or labetalol infusion or a combination of both for BP control, were included for analysis. Both the agents were found to be equally safe and effective for $\mathrm{BP}$ control in $\mathrm{ICH}$ during the initial admission hours.

\section{Choice of Antihypertensive Agent in Patients with ICH after Discharge}

It is clear that patients with ICH have a high risk of recurrence of ICH over the short term as well as long term. Therefore, $\mathrm{BP}$ control is of paramount importance. Antihypertensive medication should be started at the earliest, and all patients at discharge from hospital must be on antihypertensive agents. There is no specific antihypertensive agent that is preferentially used in this population. The choice depends on other factors, such as age and comorbid conditions (diabetes mellitus, renal insufficiency, cardiac disease, etc) and can be decided as in general population with hypertension.

In progress trial, perindopril-based lowering of $\mathrm{BP}$ reduced the risk of ICH recurrence by $46 \%$ over a mean follow-up of 3.9 years. ${ }^{25}$

The latest American Heart Association/American Stroke Association guidelines recommend a target BP of $<130 / 80 \mathrm{~mm} \mathrm{Hg}$ after $\mathrm{ICH}$, in order to reduce the risk of ICH recurrence. In SPS3 trial, the rate of ICH was reduced by $63 \%$ in the lower target group (systolic $\mathrm{BP}<130 \mathrm{~mm} \mathrm{Hg}$ ). ${ }^{26}$

\section{CONCLUSION}

Hypertension is an important risk factor for incident as well as recurrent hemorrhagic stroke. Elevated BP in the acute phase of ICH is associated with worse functional and neurological outcomes. Rapid lowering of BP is needed if systolic BP > $180 \mathrm{~mm} \mathrm{Hg}$ in the early period after ICH. Aggressive control with antihypertensive medications is needed for long-term duration to reduce the risk of $\mathrm{ICH}$ recurrence.

\section{REFERENCES}

1. Ariesen MJ, Claus SP, Rinkel GJ, Algra A. Risk factors for intracerebral hemorrhage in the general population: a systematic review. Stroke 2003 Aug; 34(8):2060-2065.

2. Leppälä JM, Virtamo J, Fogelholm R, Albanes D, Heinonen OP. Different risk factors for different stroke subtypes: association of blood pressure, cholesterol, and antioxidants. Stroke 1999 Dec; 30(12):2535-2540.
3. Suh I, Jee SH, Kim HC, Nam CM, Kim IS, Appel LJ. Low serum cholesterol and haemorrhagic stroke in men: Korea Medical Insurance Corporation Study. Lancet 2001 Mar; 357(9260):922-925.

4. Martí-Fàbregas J, Prats-Sánchez L, Martínez-Domeño A, Camps-Renom P, Marín R, Jiménez-Xarrié E, Fuentes B, Dorado L, Purroy F, Arias-Rivas S, et al. The H-ATOMIC Criteria for the Etiologic Classification of Patients with Intracerebral Hemorrhage. PLoS One 2016 Jun; 11:e0156992.

5. Liu J, Wang D, Lei C, Xiong Y, Yuan R, Hao Z, Tao W, Liu M. Etiology, clinical characteristics and prognosis of spontaneous intracerebral hemorrhage in children: a prospective cohort study in China. J Neurol Sci 2015 Nov; 358(1-2):367-370.

6. Johansson BB. Hypertension mechanisms causing stroke. Clin Exp Pharmacol Physiol 1999 Jul;26(7):563-565.

7. Zafar A, Khan FS. Clinical and radiological features of intracerebral hemorrhage in hypertensive patients. J Pak Med Assoc 2008 Jul;58(7):356-358.

8. Wiener HL, Cooper PR. The management of spontaneous intracerebral hemorrhage. Contemp Neurosurg 1992;14(21):1-8.

9. Cappellari M, Zivelonghi C, Moretto G, Micheletti N, Carletti M, Tomelleri G, Bovi P. The etiologic subtype of intracerebral hemorrhage may influence the risk of significant hematoma expansion. J Neurol Sci 2015 Dec; 359(1-2):293-297.

10. Lord AS, Gilmore E, Choi HA, Mayer SA; VISTA-ICH Collaboration. Time course and predictors of neurological deterioration after intracerebral hemorrhage. Stroke 2015 Mar; 46(3):647-652.

11. Urday S, Beslow LA, Dai F, Zhang F, Battey TW, Vashkevich A, Ayres AM, Leasure AC, Selim MH, Simard JM, et al. Rate of perihematomal edema expansion predicts outcome after intracerebral hemorrhage. Crit Care Med 2016 Apr;44(4):790-797.

12. Kobayashi J, Koga M, Tanaka E, Okada Y, Kimura K, Yamagami H, Okuda S, Hasegawa Y, Shiokawa Y, Furui E, et al; SAMURAIStudy Investigators. Continuous antihypertensive therapy throughout the initial 24 hours of intracerebral hemorrhage: the stroke acute management with urgent risk-factor assessment and improvement-intracerebral hemorrhage study. Stroke 2014 Mar; 45(3):868-870.

13. Fogelholm R, Avikainen S, Murros K. Prognostic value and determinants of first-day mean arterial pressure in spontaneous supratentorial intracerebral hemorrhage. Stroke 1997 Jul; 28(7):1396-1400.

14. Anderson CS, Heeley E, Huang Y, Wang J, Stapf C, Delcourt C, Lindley R, Robinson T, Lavados P, Neal B, et al; INTERACT 2 Investigators. Rapid blood-pressure lowering in patients with acute intracerebral hemorrhage. N Engl J Med 2013 Jun; 368(25):2355-2365.

15. Qureshi Al, Palesch YY, Barsan WG, Hanley DF, Hsu CY, Martin RL, Moy CS, Silbergleit R, Steiner T, Suarez JI, et al; ATACH-2 Trial Investigators and the Neurological Emergency Trials Network. N Engl J Med 2016 Sep; 375(11): 1033-1043.

16. Jusufovic M, Sandset EC, Bath PM, Berge E, Scandinavian Candesartan Acute Stroke Trial Study Group. Blood pressurelowering treatment with candesartan in patients with acute hemorrhagic stroke. Stroke 2014 Nov; 45(11):3440-3442.

17. Potter JF, Robinson TG, Ford GA, Mistri A, James M, Chernova J, Jagger C. Controlling hypertension and hypotension immediately post-stroke (CHHIPS): a randomised, placebo-controlled, double-blind pilot trial. Lancet Neurol 2009 Jan;8(1):48-56. 
18. Robinson TG, Potter JF, Ford GA, Bulpitt CJ, Chernova J, Jagger C, James MA, Knight J, Markus HS, Mistri AK, et al; COSSACS Investigators. Effects of antihypertensive treatment after acute stroke in the Continue or Stop Post-Stroke Antihypertensives Collaborative Study (COSSACS): a prospective, randomised, open, blinded-endpoint trial. Lancet Neurol 2010 Aug;9(8):767-775.

19. Bath PM, Woodhouse L, Scutt P, Krishnan K, Wardlaw JM, Bereczki D, Sprigg N, Berge E, Beridze M, Caso V, et al; ENOS trial investigators. Efficacy of nitric oxide, with or without continuing antihypertensive treatment, for management of high blood pressure in acute stroke (ENOS): a partial-factorial randomised controlled trial. Lancet 2015 Feb; 385(9968): 617-628.

20. Hemphil JC 3rd, Greenberg SM, Anderson CS, Becker K, Bendok BR, Cushman M, Fung GL, Goldstein JN, Loch Macdonald R, Mitchell PH, et al; American Heart Association Stroke Council; Council on Cardiovascular and Stroke Nursing; Council on Clinical Cardiology. Guidelines for the management of spontaneous intracerebral hemorrhage: a guideline for healthcare professionals from the American Heart Association/American Stroke Association. Stroke 2015 Jul; 46(7):2032-2060.

21. Schmidt LB, Goertz S, Wohlfahrt J, Melbye M, Munch TN. Recurrent intracerebral hemorrhage: associations with comorbidities and medicine with antithrombotic effects. PLoS One 2016 Nov;11(11):e0166223.
22. Biffi A, Anderson CD, Battey TW, Ayres AM, Greenberg SM, Viswanathan A, Rosand J. Association between blood pressure control and risk of recurrent intracerebral hemorrhage. JAMA 2015 Sep; 314(9):904-912.

23. Koga M, Toyoda K, Naganuma M, Kario K, Nakagawara J, Furui E, Shiokawa Y, Hasegawa Y, Okuda S, Yamagami H, et al; Stroke Acute Management with Urgent Risk-factor Assessment and Improvement (SAMURAI) Study Investigators. Nationwide survey of antihypertensive treatment for acute intracerebral hemorrhage in Japan. Hypertens Res 2009 Sep; 32(9):759-764.

24. Ortega-Gutierrez S, Thomas J, Reccius A, Agarwal S, Lantigua H, Li M, Carpenter AM, Mayer SA, Schmidt JM, Lee K, et al. Effectiveness and safety of nicardipine and labetalol infusion for blood pressure management in patients with intracerebral and subarachnoid hemorrhage. Neurocrit Care 2013 Feb;18(1):13-19.

25. Arima H, Tzourio C, Anderson C, Woodward M, Bousser MG, MacMahon S, Neal B, Chalmers J; PROGRESS Collaborative Group. Effects of perindopril-based lowering of blood pressure on intracerebral hemorrhage related to amyloid angiopathy: the PROGRESS trial. Stroke 2010 Feb;41(2): 394-396.

26. Benavente OR, Coffey CS, Conwit R, Hart RG, McClure LA, Pearce LA, Pergola PE, Szychowski JM; SPS3 Study Group. Blood-pressure targets in patients with recent lacunar stroke: the SPS3 randomised trial. Lancet 2013 Aug;382(9891):507-515. 